Journal of Computer Science 6 (10): 1159-1163, 2010

ISSN 1549-3636

(C) 2010 Science Publications

\title{
Performance Study of Ad-Hoc Reactive Routing Protocols
}

\author{
Pallavi Khatri, Monika Rajput, Alankar Shastri and Keshav Solanki \\ Department of Computer Science and Information Technology, \\ Institute of Technology and Management, \\ NH-75, Opp. Sithouli Railway Station, Gwalior, MP, India
}

\begin{abstract}
Problem statement: In this study, an attempt had been made to compare the performance of the reactive ad-hoc routing protocols using OPNET modeler with respect to increasing number of nodes in the network. Approach: In present study, we compared various reactive routing protocols such as Ad hoc On-demand Distance Vector (AODV), Dynamic Source Routing (DSR) and Temporally Ordered Routing Algorithm (TORA), on the basis of their throughput by increasing number of nodes in the network. Results: Comparative study of routing protocols on the basis of throughput of a network on the basis of number of increasing nodes in the network. Conclusion/Recommendations: Three routing protocols are being studied on the basis of the throughput of the network with respect to the number of increasing nodes in the network and had been concluded that TORA performs better for if the number of nodes in a network are increased.
\end{abstract}

Keywords: MANET, AODV, DSR, TORA, OPNET

\section{INTRODUCTION}

A Mobile Ad-hoc Network (MANET) is a multi hop wireless network formed by a group of mobile nodes that have wireless capabilities. MANET is a collection of wireless nodes that dynamically create a wireless network among them without any infrastructure (Sheltami and Mouftah, 2003). Ad-hoc is a communication mode that allows computers to directly communication with each other without a router. In Latin, ad-hoc means "for this" meaning "for this special purpose". In ad hoc networks, nodes do not start out familiar with the topology of their networks; instead, they have to discover it. The basic idea is that a new node may announce its presence and should listen for announcements broadcast by its neighbors. Each node learns about nodes nearby and how to reach them and may announce that it, too, can reach them. Ad-hoc network can be sub-divided into two classes. In Static ad-hoc network the positions of a node may not change once it has become part of the network. Ex-Rooftop networks. And in Mobile ad-hoc network is Sometimes called a Mobile Mesh N/W, IS a self configuring N/W of mobile devices connected by wireless links. Each device in a MANET is free to move independently in any direction. The primary challenge in building a MANET is equipping each device to continuously maintain the information required to properly route traffic. MANETs are a kind of wireless ad-hoc network. As the network topology is dynamic a routing protocol is needed to support the proper functionality of the network. Some of the prominent and promising among them are Ad hoc On-demand and Distance Vector (AODV), Dynamic Source Routing (DSR), TORA and Destination-Sequenced Distance Vector (DSDV). This study provides a comparative study through simulation of three routing protocols (DSR, AODV, TORA) for mobile ad-hoc networks using OPNET modeler. The main objective of this study is to create a choice guide of routing protocol for a given network scenario, based on the relative performance of the protocol under different scenarios. The study briefly describes the routing protocols; the simulation tool used for this work, simulation environment, brief discussion on simulation mode, results and concluding remarks.

Ad-hoc routing protocols: As the topology of Ad-hoc networks is dynamic, the need of various routing protocols is developed. Main features of the three protocols AODV, DSR, TORA are discussed here and they are studied using OPNET simulator (Jiang et al., 1999).

An ad hoc routing protocol is a convention, or standard, that controls how nodes decide which way to route packets between computing devices in a mobile ad-hoc network.

Corresponding Author: Pallavi Khatri, Department of Computer Science and Information Technology,

Institute of Technology and Management, NH-75, Opp. Sithouli Railway Station, Gwalior, MP, India 
On demand/reactive routing protocol: On-demand routing protocols were designed to reduce the overheads in proactive protocols by maintaining information for active routes only. This means that routes are determined and maintained for nodes that require sending data to a particular destination. Route discovery usually occurs by flooding a route request packets through the network. When a node with a route to the destination (or the destination itself) is reached a route reply is sent back to the source node using link reversal if the route request has traveled through bidirectional links or by piggy-backing the route in a route reply packet via flooding. Reactive protocols can be classified into two categories: source routing and hop-by-hop routing. In source routed on-demand protocols, each data packets carry the complete source to destination address. Therefore, each intermediate node forwards these packets according to the information kept in the header of each packet. This means that the intermediate nodes do not need to maintain up-to-date routing information for each active route in order to forward the packet towards the destination. Furthermore, nodes do not need to maintain neighbor connectivity through periodic beaconing messages. The major drawback with source routing protocols is that in large networks they do not perform well. This is due to two main reasons; firstly as the number of intermediate nodes in each route grows, then so does the probability of route failure. The advantage of this strategy is that routes are adaptable to the dynamically changing environment of MANETs, since each node can update its routing table when they receiver fresher topology information and hence forward the data packets over fresher and better routes. Under this category Dynamic Source Routing (DSR) protocol requires each packet to carry the full address (every hop in the route), from source to the destination. Ad hoc Ondemand Distance Vector (AODV) routing protocol is based on DSDV and DSR algorithm. It uses the periodic beaconing and sequence numbering procedure of DSDV and a similar route discovery procedure as in DSR. Temporally Ordered Routing Algorithm (TORA) routing protocol is based on the LMR protocol. It uses similar link reversal and route repair procedure as in LMR and also the creation of a DAGs: these routing protocols are briefly described below.

Ad-hoc On Demand Distance Vector (AODV): AODV (Perkins et al., 2003; Chaudhry et al., 2005) is an reactive (On-demand routing protocol) with small delay. Since it is an "On-demand" routing protocol, the rotes are established only when needed to reduce traffic load. AODV supports the Unicast, Broadcast and
Multicast scheme. The Count-To-Infinity and loop problem is solved with sequence numbers and the registration of the costs. In AODV every hop has the constant cost of one. The routes age very quickly in order to accommodate the movement of the mobile nodes. Link breakages can locally be repaired very efficiently. AODV is a modification of the DSDV algorithm. When a source node desires to establish communication session, it initiates a path discovery process to locate the other node. The main advantage of AODV protocol is that routes are established on demand and destination sequence numbers are used to find the latest route to the destination. The connection setup delay is less. The HELLO messages supporting the routes maintenance are range-limited, so they do not cause unnecessary overhead in the network.

Dynamic Source Routing (DSR): The Dynamic Source Routing protocol (DSR) (Johnson et al., 1999) is a simple and efficient routing protocol designed specifically for use in multi-hop wireless Ad- networks of mobile nodes. DSR allows the network to be completely self-organizing and self-configuring, without the need for any existing network infrastructure or administration. The protocol is composed of the two main mechanisms of "Route Discovery" and "Route Maintenance", which work together to allow nodes to discover and maintain routes to arbitrary destinations in the ad hoc network. However, this protocol has a number of advantages over routing protocols such as AODV, LMR and TORA and in small to moderately size networks (perhaps up to a few hundred nodes), this protocol may perform better. An advantage of DSR is that nodes can store multiple routes in their route cache, which means that the source node can check its route cache for a valid route before initiating route discovery and if a valid route is found there is no need for route discovery. This is very beneficial in network with low mobility. Since they routes stored in the route cache will be valid longer. Another advantage of DSR is that it does not require any periodic beaconing (or hello message exchanges), therefore nodes can enter sleep node to conserve their power. This also saves a considerable amount of bandwidth in the network.

\section{Temporally Ordered Routing Algorithm (TORA):} Temporally-Ordered Routing Algorithm (TORA) (Park and Corson, 1997a; 1997b) is a distributed protocol designed to be highly adaptive so it can operate in a dynamic network. For a given destination, TORA uses a somewhat arbitrary "height" parameter to determine the direction of a link between any two nodes. As a consequence of this multiple routes are often present for a given destination, but none of them are necessarily the 
shortest route The TORA routing protocol is based on the LMR protocol. It uses similar link reversal and route repair procedure as in LMR and also the creation of a DAGs, which is similar to the query/reply process used in LMR. Therefore, it also has the same benefits as LMR. The advantage of TORA is that it has reduced the far-reaching control messages to a set of neighboring nodes, where the topology change has occurred. Another advantage of TORA is that it also supports multicasting; however this is not incorporated into its basic operation. TORA can be used in conjunction with Lightweight Adaptive Multicast Algorithm (LAM) to provide multicasting. The disadvantage of TORA is that the algorithm may also produce temporary invalid routes as in LMR.

Simulation environment: All scenarios have been modeled and evaluated using OPNET (OPNET Technologies, 2005; Cavin et al., 2002; Camp et al., 2002). Figure 1 shows a sample network created with 20 nodes, one static FTP server, application configuration for the network in which File Transfer Protocol (FTP) has been chosen as an application, Profile configuration for the network and mobility configuration for the nodes which would be deciding the mobility pattern of the nodes in the network.

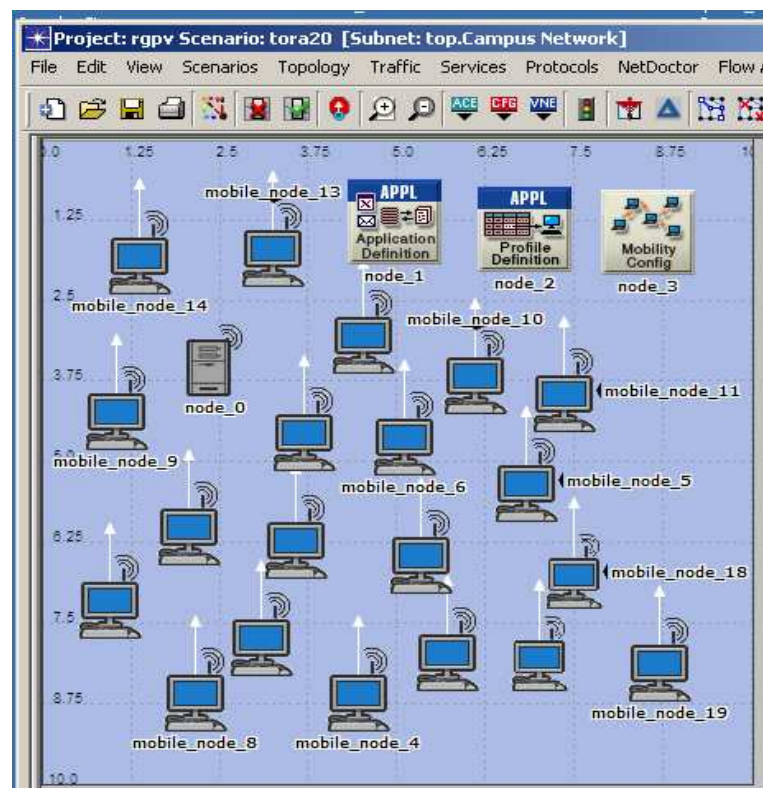

Fig. 1: MANET scenario

Table 1: Main characteristic of scenario

\begin{tabular}{ll}
\hline Statistic & Value \\
\hline Scenario size & $10 \times 10 \mathrm{~km}$ \\
Simulation time & $1 \mathrm{~h}$ \\
Nodes & $5,10,20$ \\
802.11 data rate & $11 \mathrm{mbps}$ \\
\hline
\end{tabular}

Figure 1 depicts a network with 20 mobile nodes whose behavior has to be analyzed when nodes move in the network with respect to time to determine the effecting features of each protocol. In order to evaluate the performance of a generic scenario in ad-hoc networking, when analyzing mobile networks, modeling the movement of the set of nodes forming a MANET is essential. Random waypoint model (Johnson and Maltz, 1996) of mobility has been studied. The Random Waypoint model has been selected to be used in all simulations presented in this document. Using Random Waypoint model, nodes go moving until they arrive at a random destination calculated by the algorithm. Once there, they get still for a period of time, called the pause interval. Once passed the pause interval, anew movement is calculated by the algorithm, with a random direction and speed.

Simulation model: Main characteristics of the scenarios maintained are depicted in the Table 1.

Traffic modeling: Our simulation environment consist of 20 wireless nodes forming an ad-hoc network, moving in the proximity over about $10 \times 10 \mathrm{~km}$ flat space for about $1 \mathrm{~h}$ of simulated time.

Performance matrices: The parameters on basis of which the protocols are evaluated are the default parameter of the protocols. There are number of metrics on the basis of which one can compare between these three protocols. We used throughput for our simulation design and analysis purpose.

Throughput (bps): Represents the total number of bits forwarded to higher layers per second.

\section{MATERIALS AND METHODS}

OPNET modeler: OPNET Modeler is commercial network simulation environment for network modeling and simulation. It allows the users to design and study communication networks, devices, protocols and applications with flexibility and scalability. It simulates the network graphically and its graphical editors mirror the structure of actual networks and network components. The users can design the network model visually. The modeler uses object-oriented modeling approach. The nodes and protocols are modeled as classes with inheritance and specialization. The development language is $\mathrm{C}$. It provides a variety of toolbox to design, simulate and analyze a network topology. We use MANET Toolbox to simulate our hypothetical Ad hoc network. We used MANET_Station, RX Group and Mobility configuration in our simulation model. MANET_station can be used to set routing algorithm, traffic generation parameter. Moreover, we can also set parameters specific to routing algorithm. 


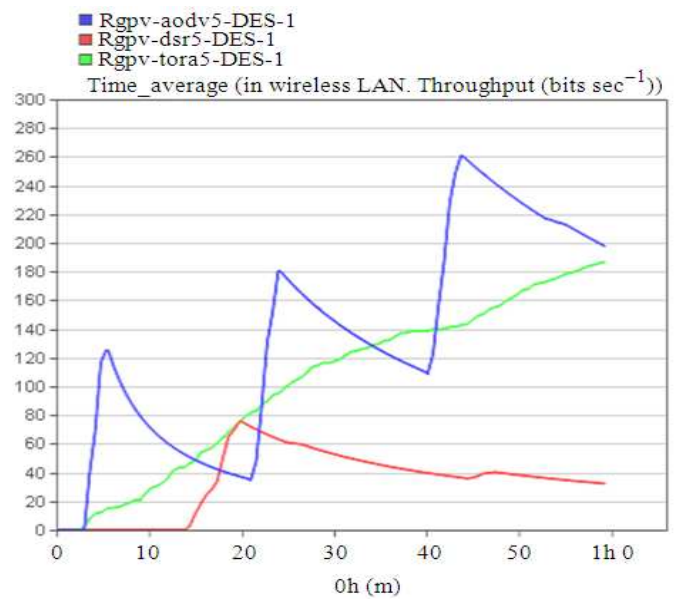

Fig. 2: Simulation time Vs throughput (nodes $=5$ )

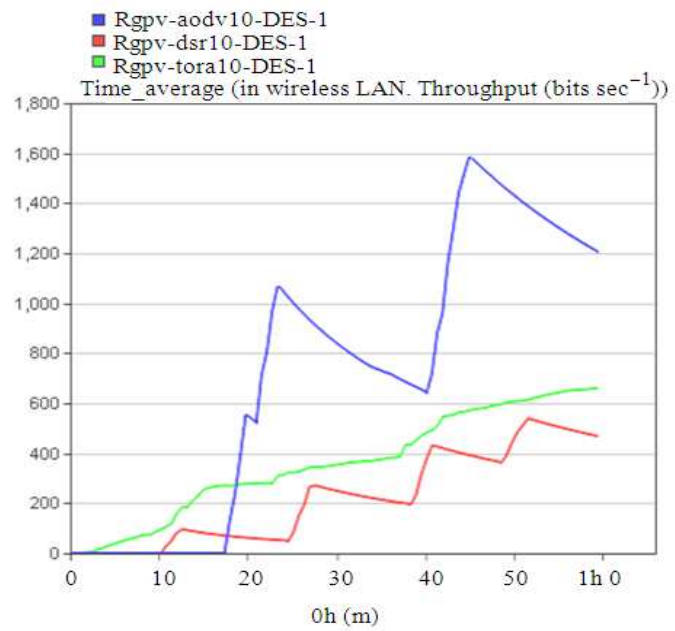

Fig. 3: Simulation time Vs throughput $($ nodes $=10)$

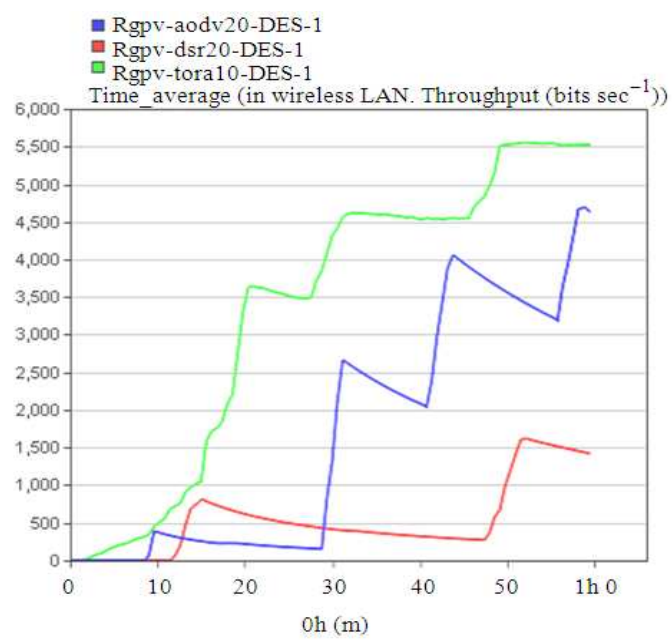

Fig. 4: Simulation time Vs throughput $($ nodes $=20)$

\section{RESULTS AND DISCUSSION}

Figure 2-4 depicts the throughput of a network with respect to total simulation time when the number of nodes in the network is 5, 10 and 20 respectively.

As seen in Fig. 2-4 the throughput of AODV increases sharply up to a certain level and decreases abruptly for some time and then increases again. Initially it takes a bit of time to discover the route and then start sending the packets. Since it does not have multiple entries in its routing table, AODV has to do the route discovery again and again at regular interval. As we increase the number of nodes the throughput of AODV also increases. In case of DSR, initially it takes much time as it has to make multiple entries of routes gathered after route discovery in its routing table. After route discovery its throughput increases uniformly but less then AODV and TORA. Whereas in case of TORA, the throughput increases continuously. TORA makes a Direct Allocation Graph (DAG) of all the nodes then start sending packets. TORA does not engage in the route discovery again and again as it already have a DAG of all the nodes in the network. The throughput of TORA increases with increase in number of nodes.

\section{CONCLUSION}

We have analyzed the performance of three reactive routing protocols for ad-hoc networks on the above mentioned matrices and concluded that performance of TORA is better for dense networks. The AODV is better for moderately dense networks where as the DSR performs well in sparse networks.

\section{REFERENCES}

Camp, T., J. Boleng and V. Davies, 2002. A survey of mobility models for ad hoc network research. Wireless Commun. Mobile Comput., 2: 483-502.

Cavin, D., Y. Sasson and A. Schiper, 2002. On the accuracy of MANET simulators. Proceedings of the 2nd ACM International Workshop on Principles of Mobile Computing, Oct. 30-31, ACM Press, Toulouse, France, pp: 38-43.

Chaudhry, S.R., A. Al-Khwildi, Y. Casey, H. Aldelou and H.S. Al-Raweshidy, 2005. A performance comparison of multi on demand routing in wireless ad hoc networks. Proceedings of the IEEE International Conference on Wireless and Mobile Computing, Networking Communication, Aug. 2224, IEEE Xplore Press, USA., pp: 9-16. 
Jiang, M., J. Li and Y.C. Tay, 1999. Cluster based routing protocol. IETF Draft. http://wiki.uni.lu/secanlab/Cluster+Based+Routing+Protocol.html

Johnson, D.B. and D.A. Maltz, 1996. Dynamic Source Routing in Ad Hoc Wireless Networks. In: Mobile Computing, Imielinski, T. and H.F. Korth (Eds.). Springer, US., ISBN: 978-0-585-29603-6, pp: 153-181.

Johnson, D.B., D.A. Maltz and J. Broch, 1999. DSR: The Dynamic Source Routing Protocol for Multihop Wireless Ad Hoc Networks. In: In Ad Hoc Networking, Perkins, C.E. (Ed.). AddisonWesley, USA., pp: 139-172.

OPNET Technologies, 2005. OPNET: Making networks and applications perform. OPNET Technologies Inc.

Park, V.D. and M.S. Corson, 1997a. A highly adaptive distributed routing algorithm for mobile wireless networks. Proceedings of the 16th Annual Joint Conference of the IEEE Computer and Communications Societies, Apr. 9-11, IEEE Computer Society, Washington DC., USA., pp: 1-9.
Park, V.D. and M.S. Corson, 1997b. Temporallyordered routing algorithm. University of Luxembourg. http://wiki.uni.lu/secanlab/Temporally-Ordered+Routing+Algorithm.html

Perkins, C.E., E. Belding-Royer and S.R. Das, 2003. Ad Hoc on-demand distance vector routing. Network Working Group. http://moment.cs.ucsb.edu/pub/rfc3561.txt

Sheltami, T. and H.T. Mouftah, 2003. An efficient energy aware clusterhead formation infrastructure protocol for MANETs. Proceeding of the 8th IEEE Symposium on Computers and Communication, June 30-July 3, IEEE Computer Society, Washington DC., USA., pp: 203-208. 